\title{
Optimal Distributed Beamforming in Relay Networks with Common Interference
}

\author{
Krishna S. Gomadam, Syed A. Jafar \\ Electrical Engineering and Computer Science \\ University of California, Irvine, CA 92697-2625 \\ Email: \{kgomadam, syed $\} @$ uci.edu
}

\begin{abstract}
In wireless relay networks, noise at the relays can be correlated possibly due to common interference or noise propagation from preceding hops. In this work we consider a parallel relay network with noise correlation. For the relay strategy of amplify-and-forward (AF), we determine the optimal rate maximizing relay gains when correlation knowledge is available at the relays. The effect of correlation on the performance of the relay networks is analyzed for the cases where full knowledge of correlation is available at the relays and when there is no knowledge about the correlation structure. Interestingly we find that, on the average, noise correlation is beneficial regardless of whether the relays know the noise covariance matrix or not. However, the knowledge of correlation can greatly improve the performance. Typically, the performance improvement from correlation knowledge increases with the relay power and the number of relays. With perfect correlation knowledge the system is capable of canceling interference if the number of interferers is less than the number of relays.
\end{abstract}

\section{INTRODUCTION}

Wireless mesh networks where information is transferred via multiple hops and routes provide significant throughput enhancement and have been the focus of much recent work [1], [2]. Various relay strategies have been studied in literature [3]-[5]. Among these strategies, amplify and forward has been found to be highly suitable for parallel relay networks for its ability to pass on soft information [6], [7]. For an AF relay network, the relay design involves optimizing the relay amplification factors to maximize performance. Previous work pertaining to relay optimization for AF relay networks includes both single-user relaying [8]-[11] and multi-user relaying [2], [12].

Most work in the literature assumes independent noise at the relay terminals. However, noise correlation between nodes occurs in wireless relay networks due to several reasons. In this paper we explore the effect of noise correlation for the following two models.

1) Common Interference Model

Due to the broadcast nature of wireless networks, the relays are exposed to a set of common interferers resulting in correlated noise at the nodes.

2) Noise propagation with multi-hop AF relaying

For example, in a three-hop AF network, every relay in the second hop observes a linear combination of noise from the relays of the preceding stage. This clearly results in correlated noise at the second-hop relays.

Both the above models are of considerable practical importance. The natural question is whether the relays can exploit the correlation structure to improve performance. In practice, learning correlation may result in network overheads. Whether such overheads are justified depends on the potential advantage of learning correlation. Thus, our goal in this work is to estimate the improvement in performance when perfect correlation knowledge is available at the relays. The optimal relay design in this case will have the following two objectives:

1) Increase signal power: The relay gains can be designed such that the copies of desired signal adds up in phase.

2) Reduce interference power: The relay gains may be chosen such that the common interference terms add out of phase.

In general, these objectives cannot be achieved simultaneously and the relay design can be expected to be a trade-off between the two objectives.

\section{A. Results}

The main results of this paper are summarized below.

1) We obtain closed form solutions for the optimal relay amplification vector and the maximum sum rate for the network with correlated noise at the relays. This result generalizes the single-user relay optimization in [8], [9] and the multi-source relay optimization in [2] both of which assume independent and identically distributed (i.i.d.) noise at the relays.

2) We find that correlation, on the average, is always beneficial regardless of the presence or absence of correlation knowledge at the relays. This is true irrespective of the channel state information (CSI) at the relays.

3) We study the benefits of exploiting noise correlation at the relays. We compare the maximum sum rate without correlation knowledge (relays use optimal amplification factors based on uncorrelated noise assumption even if noise is correlated) versus capacity with correlation knowledge. The following key questions are answered: Does correlation help? Is the correlation knowledge more (or less) helpful as the number of relays increases? What is the effect of correlation as the power at the relays increases, and when the first hop/second hop becomes stronger? We also provide asymptotic results to characterize the impact of relay noise correlation.

\section{System Model}

We consider a multi-source parallel relay network as shown in Fig. 1. In this model, $L$ source nodes wish to communicate to a common destination through a set of $N$ relays. The data transmission takes place in two time slots. In the first slot, 
the sources transmit to the relays and in the second slot, all the relays simultaneously forward their received signal to the destination. Note that there is no direct link between the source and the destination. All the nodes are equipped with a single antenna. Here each source has power $\left.P_{k}\right\}_{k=1}^{L}$ and the

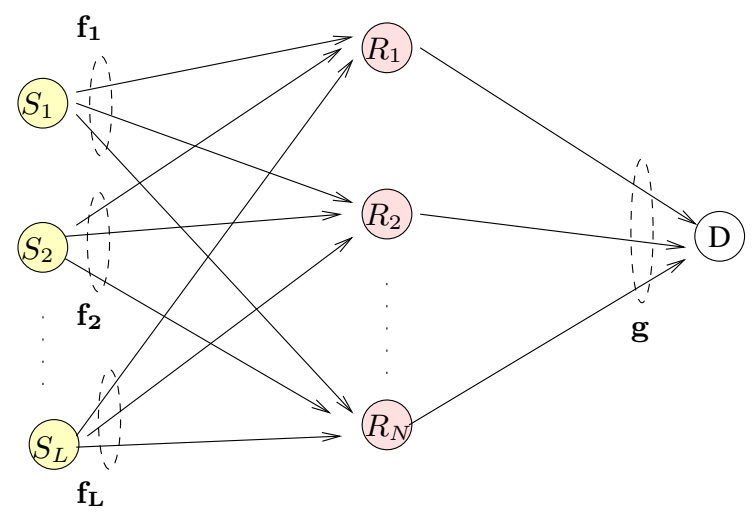

Fig. 1. Two Hop Multi-Source Parallel Relay Network

relays have a sum power constraint of $P_{R}$. The relay received symbols during the first time slot are given by

$$
\mathbf{r}=\sum_{k=1}^{L} \mathbf{f}_{\mathbf{k}} x_{k}+\mathbf{n}_{\mathbf{R}}
$$

where $\mathbf{f}_{\mathbf{k}}$ represents the $N \times 1$ channel between the $k^{\text {th }}$ source and the relays, $x_{k}$ is the symbol transmitted by the $k^{\text {th }}$ source with power $\mathcal{E}\left[\left|x_{k}\right|^{2}\right]=P_{k}$. The elements of $\mathbf{n}_{\mathbf{R}}$ are AWGN with the covariance matrix given by $\mathbf{K}=\mathcal{E}\left[\mathbf{n}_{\mathbf{R}} \mathbf{n}_{\mathbf{R}}^{\dagger}\right]$. In the second slot, the relay $\left.R_{i}\right\}_{i=1}^{N}$ scales its received signal with a complex scaling factor $d_{i}$ and transmits to the destination. We collect the relay gain factors $d_{i}$ in an $N \times 1$ vector $\mathbf{d}$. Then the received signal at the destination can be expressed as

$$
y=\sum_{k=1}^{L} \mathbf{d}^{\mathbf{T}} \mathbf{G f}_{\mathbf{k}} x_{k}+\mathbf{d}^{\mathbf{T}} \mathbf{G n}_{\mathbf{R}}+n_{D}
$$

where $\mathbf{G}=\operatorname{diag}(\mathbf{g})$ and $n_{D} \sim \mathcal{C N}(0,1)$ is additive white Gaussian noise (AWGN). Further, we assume that there is no correlation between destination noise $n_{D}$ and the relay noise vector $\mathbf{n}_{\mathbf{R}}$ as these noise processes occur at two different time slots. The relays have a sum power constraint which can be expressed as

$$
\mathbf{d}^{\mathbf{T}}\left[\left(\sum_{k=1}^{L} \mathbf{f}_{\mathbf{k}} \mathbf{f}_{\mathbf{k}}^{\dagger} P_{k}+\mathbf{K}\right) \odot \mathbf{I}\right] \mathbf{d}^{*}=P_{R}
$$

where $\odot$ represents the element-wise multiplication of two matrices. The achievable sum-rate for this network is given by $^{1}$

$$
R_{\sum}=\log \left(1+\frac{\sum_{k=1}^{L}\left|\mathbf{d}^{\mathbf{T}} \mathbf{G f}_{\mathbf{k}}\right|^{2} P_{k}}{\mathbf{d}^{\mathbf{T}} \mathbf{G} \mathbf{K} \mathbf{G}^{*} \mathbf{d}^{*}+1}\right) .
$$

We seek to maximize the sum rate of the network with respect to the amplification vector $\mathbf{d}$ subject to the sum power constraint at the relays. The following theorem provides the optimal relay design and the maximum achievable sum rate.

\footnotetext{
${ }^{1}$ Gaussian inputs are optimal for AF relaying.
}

Theorem 1: The maximum achievable sum rate in a twohop multi-source parallel AF relay network with noise correlation is given by $R^{\circ}=\log \left(1+\mathrm{SNR}^{\circ}\right)$ where

$$
\mathrm{SNR}^{\circ}=P_{R} \lambda_{\max }\left[\mathbf{A}^{-\mathbf{1}} \mathbf{B}\right]
$$

and the optimal relay amplification vector $\mathbf{d}_{\Sigma}^{\circ}$ is

$$
\mathbf{d}_{\mathbf{\Sigma}}^{\circ}=\kappa\left(\mathbf{v}_{\max }\left[\mathbf{A}^{-\mathbf{1}} \mathbf{B}\right]\right)^{*}
$$

where

$$
\mathbf{A}=\mathbf{K} \odot \mathbf{g g}^{\dagger} P_{R}+\sum_{k=1}^{L} \mathbf{f}_{\mathbf{k}} \mathbf{f}_{\mathbf{k}}^{\dagger} P_{k} \odot \mathbf{I}+\mathbf{K} \odot \mathbf{I}
$$

and $\mathbf{B}=\sum_{k=1}^{L}\left(\mathbf{f}_{\mathbf{k}} \odot \mathbf{g}\right)\left(\mathbf{f}_{\mathbf{k}} \odot \mathbf{g}\right)^{\dagger} P_{k}$. Here $\lambda_{\max }(\mathbf{A})$ and $\mathbf{v}_{\max }(\mathbf{A})$ denote the principal eigen-value and principal eigenvector of $\mathbf{A}$ respectively, and $\kappa$ ensures compliance with the relay power constraint.

The proof is available in [13]. For the single user case $(L=1)$, let $P=P_{1}$ and $\mathbf{f}=\mathbf{f}_{\mathbf{1}}$. Now the optimal relay amplification vector $\mathbf{d}^{o}$ is

$$
\mathbf{d}^{o}=\kappa\left(\mathbf{A}^{-1}(\mathbf{f} \odot \mathbf{g})\right)^{*}
$$

and the maximum SNR is given by

$$
\mathrm{SNR}^{o}=P P_{R}(\mathbf{f} \odot \mathbf{g})^{\dagger} \mathbf{A}^{-\mathbf{1}}(\mathbf{f} \odot \mathbf{g})
$$

where

$$
\mathbf{A}=\mathbf{K} \odot \mathbf{g g}^{\dagger} P_{R}+\mathbf{f f}^{\dagger} P \odot \mathbf{I}+\mathbf{K} \odot \mathbf{I} .
$$

Note that $\mathbf{A}$ is not a diagonal matrix. Therefore the optimal relay gain may not co-phase the input signal as some part of noise can be canceled with gain adjustments. For the remainder of this work we will focus on the single user scenario to gain insights into the impact of relay noise correlation.

\section{Does CoRRElation Help?}

In this section, we address the following important questions:

1) Does correlation help when the relays know the covariance matrix?

2) Does correlation hurt when the relays are unaware of it? To answer the first question we compare the following two scenarios.

- The noise is correlated and relays are aware of the correlation. (Scheme-11)

- The noise is uncorrelated. (Scheme-00)

To answer the second question we compare the following two scenarios.

- The noise is correlated and relays are unaware of the correlation. (Scheme-10)

- The noise is uncorrelated. (Scheme-00)

If Scheme-11 outperforms Scheme-00 then we can say that correlation helps if the relays are aware of it. Similarly, if Scheme-00 outperforms Scheme-10 then we can say that correlation hurts when the relays are unaware of it.

\section{A. Benchmark Schemes}

1) Relays with uncorrelated noise: Scheme-00: This setup has been commonly studied in literature [2], [8]-[10]. The system model consists of a two hop parallel relay network with independent noise at the relays. The noise covariance 
matrix, which is diagonal, is given by $\mathbf{K} \odot \mathbf{I}$. For a sum power constraint at the relays, the optimum relay amplification vector is found in [8], [9]. The result can also be obtained from Theorem 1 by replacing $\mathbf{K}$ with $\mathbf{K} \odot \mathbf{I}$. The achievable SNR is given by

$$
\mathrm{SNR}_{00}=P P_{R}(\mathbf{f} \odot \mathbf{g})^{\dagger}\left[\left(\mathbf{G K G} \mathbf{G}^{\dagger} P_{R}+\mathbf{f f}^{\dagger} P+\mathbf{K}\right) \odot \mathbf{I}\right]^{-1}(\mathbf{f} \odot \mathbf{g}) .
$$

A special case of Scheme-00 is where the relay noise terms are independent and identically distributed. Since we require the trace of the noise covariance matrix to be equal in all the schemes, the covariance matrix for this scheme will be $\mathbf{K}_{\text {iid }}=\frac{\operatorname{Tr}(\mathbf{K})}{N} \mathbf{I}$. We denote this model as Schemeiid indicating that the relay noise terms are i.i.d. Again, the optimal relay amplification vector for this model can be obtained from Theorem 1 by replacing $\mathbf{K}$ with $\frac{\operatorname{Tr}(\mathbf{K})}{N} \mathbf{I}$. The resulting SNR is found as

$\mathrm{SNR}_{\mathrm{iid}}=P P_{R}(\mathbf{f} \odot \mathbf{g})^{\dagger}\left[\left(\frac{\operatorname{Tr}(\mathbf{K}) \mathbf{G} \mathbf{G}^{\dagger} P_{R}}{N}+\mathbf{f f}^{\dagger} P+\frac{\operatorname{Tr}(\mathbf{K}) \mathbf{I}}{N}\right) \odot \mathbf{I}\right]^{-1}(\mathbf{f} \odot \mathbf{g})$.

2) Relays with no correlation knowledge: Scheme-10: In this scheme, correlation is induced between the relay noise terms. However the marginals remain the same as in Scheme$00, n_{r_{i}} \sim \mathcal{C N}\left(0, \mathbf{K}_{i i}\right)$; i.e. noise at relay $R_{i}$ is AWGN with variance $\mathbf{K}_{i i}$. Note that $\mathbf{K}_{i j}$ represents the correlation between the noise terms $n_{R_{i}}$ and $n_{R_{j}}$ for $i \neq j$, and in general may not be equal to zero. Since the relays do not utilize the correlation structure, the relay amplification vector is the same as in the case of Scheme-00. The SNR achieved with the relay gain $\mathbf{D}_{10}=\operatorname{diag}\left(\mathbf{d}_{10}\right)$ where $\mathbf{d}_{10}$ is the relay amplification vector for this scheme is given by

$$
\mathrm{SNR}_{10}=\frac{\left|(\mathbf{f} \odot \mathbf{g})^{\dagger} \mathbf{D}_{10}^{-1}(\mathbf{f} \odot \mathbf{g})\right|^{2} P P_{R}}{(\mathbf{f} \odot \mathbf{g})^{\dagger} \mathbf{D}_{10}^{-1} \mathbf{A} \mathbf{D}_{10}^{-1}(\mathbf{f} \odot \mathbf{g})} .
$$

As there is no correlation knowledge at the relays, the relay operation here involves only co-phasing of the input signal and does not involve noise cancelation. It is straightforward to see that Scheme-11 will perform better than Scheme-10 as it exploits the correlation structure in designing the relay amplification factors. It is not clear whether Scheme-10 is also inferior to Scheme-00 and Scheme-iid. It is also not clear which is better among Scheme-00 and Scheme-iid. Answering these questions will provide useful insights into the impact of correlation in multi-hop AF relay networks. We address this in the rest of this section.

\section{B. Asymptotic analysis}

We compare the schemes for the two extreme cases of $P_{R} \rightarrow \infty$ and $P \rightarrow \infty$.

1) Relay Power $P_{R} \rightarrow \infty$ : At high $P_{R}$, (7) reduces to

$$
\mathrm{SNR}_{11}=\mathbf{f}^{\dagger} \mathbf{K}^{-\mathbf{1}} \mathbf{f} P \text {. }
$$

Similarly, at high $P_{R} \mathbf{D}_{\mathbf{1 0}}=\mathbf{G}(\mathbf{K} \odot \mathbf{I}) \mathbf{G}^{\dagger} P_{R}$ and $\mathbf{A}=$ $\mathrm{GKG}^{\dagger} P_{R}$. Substituting these in (11), we obtain

$$
\mathrm{SNR}_{10}=\frac{\left(\mathbf{f}^{\dagger}(\mathbf{K} \odot \mathbf{I})^{-\mathbf{1}} \mathbf{f}\right)^{\mathbf{2}} P}{\mathbf{f}^{\dagger}(\mathbf{K} \odot \mathbf{I})^{-\mathbf{1}} \mathbf{K}(\mathbf{K} \odot \mathbf{I})^{-\mathbf{1}} \mathbf{f}} .
$$

It is evident from (9) that the SNR of Scheme-11 at very high relay power is

$$
\mathrm{SNR}_{00}=P \mathbf{f}^{\dagger}(\mathbf{K} \odot \mathbf{I})^{-\mathbf{1}} \mathbf{f} .
$$

Similarly the SNR of Scheme-iid is readily obtained as

$$
\mathrm{SNR}_{\text {iid }}=\frac{\mathbf{f}^{\dagger} \mathbf{f} P}{\operatorname{Trace}(\mathbf{K}) / N} .
$$

Remark: Except for Scheme-10, all the relay schemes turn out to be a point to point SIMO channel. It must be noted that the noise at the multiple antenna receiver is correlated and the noise covariance matrix is the same as the relay noise covariance matrix of the original network. For Scheme-10, the multiple antenna receiver is unaware of the noise correlation and assumes the noise covariance matrix to be $\mathbf{K} \odot \mathbf{I}$ instead of $\mathbf{K}$.

To answer the question whether correlation hurts when there exists no knowledge of it, let us consider the difference $\mathrm{SNR}_{10}-\mathrm{SNR}_{00}$. For the simple case of $N=2$ and for real channels, we have

$$
\mathrm{SNR}_{10}-\mathrm{SNR}_{00}=\frac{-2 f_{1} f_{2} \mathbf{K}_{12}\left(f_{1}^{2} \mathbf{K}_{11}+f_{2}^{2} \mathbf{K}_{22}\right)}{f_{1}^{2} \mathbf{K}_{11}+f_{2}^{2} \mathbf{K}_{22}+2 f_{1} f_{2} \mathbf{K}_{12}}
$$

where $\mathbf{f}=\left[\begin{array}{ll}f_{1} & f_{2}\end{array}\right]^{T}$. The difference can be either positive or negative depending on the term $-2 f_{1} f_{2} \mathbf{K}_{12}$. For example, when the signals are positively correlated $\left(f_{1} f_{2} \geq 0\right)$ and the noise components are negatively correlated $\mathbf{K}_{12} \leq 0$, then correlation helps as part of noise gets canceled. Similarly when both the signal and noise components are correlated in the same direction (positive or negative) then correlation hurts as it increases the noise power. Therefore the overall effect of correlation can only be determined from the average behavior.

Theorem 2: At high relay power $P_{R}$, Scheme-10 outperforms Scheme-00 in terms of both average SNR and average rate. That is

$$
\mathbb{E}\left[\mathrm{SNR}_{10}\right] \geq \mathbb{E}\left[\mathrm{SNR}_{00}\right] \text { and } \mathbb{E}\left[\mathrm{R}_{10}\right] \geq \mathbb{E}\left[\mathrm{R}_{00}\right]
$$

where $\mathrm{R}_{10}=\log \left(1+\mathrm{SNR}_{10}\right)$ and $\mathrm{R}_{00}=\log \left(1+\mathrm{SNR}_{00}\right)$. Refer to [13] for the proof.

The above result is significant as it suggests that, in the average sense, correlation does not hurt even if correlation knowledge is not available. To determine the relationship for the rest of the cases, we take the expectation of SNR in (12), (14), and (15):

$$
\begin{aligned}
\mathbb{E}\left[\mathrm{SNR}_{11}\right] & =P \sum_{i=1}^{N} \mathbb{E}\left[\left|f_{i}\right|^{2}\right]\left(1 / \lambda_{i}\right)=P \sum_{i=1}^{N} 1 / \lambda_{i} \\
\mathbb{E}\left[\mathrm{SNR}_{00}\right] & =P \sum_{i=1}^{N} \mathbb{E}\left[\left|f_{i}\right|^{2}\right]\left(1 / \mathbf{K}_{i i}\right)=P \sum_{i=1}^{N} 1 / \mathbf{K}_{i}(18) \\
\mathbb{E}\left[\mathrm{SNR}_{\text {iid }}\right] & =\frac{P \sum_{i=1}^{N} \mathbb{E}\left[\left|f_{i}\right|^{2}\right]}{\sum_{i=1}^{N} \mathbf{K}_{i i} / N}=\frac{N P}{\sum_{i=1}^{N} \mathbf{K}_{i i} / N}
\end{aligned}
$$

where $\mathbb{E}\left[\left|f_{i}\right|^{2}\right]=1$ while $\lambda_{i}$ and $\mathbf{K}_{i i}$ are the $i^{t h}$ eigen-value and $i^{t h}$ main-diagonal term of $\mathbf{K}$ respectively. $\mathbb{E}\left[\mathbf{S N R}_{11}\right]$ is greater than $\mathbb{E}\left[\mathrm{SNR}_{00}\right]$ follows from the reason that the ordered vector containing the eigen-values of $\mathbf{K}$ majorizes the main diagonal of $\mathbf{K}$, i.e. $\lambda \succeq \mathbf{d}$. We also have $\mathbb{E}\left[\mathrm{SNR}_{00}\right] \geq \mathbb{E}\left[\mathrm{SNR}_{\text {iid }}\right]$ due to the harmonic-arithmetic mean inequality. Therefore we obtain the following relationship:

$$
\mathbb{E}\left[\mathrm{SNR}_{11}\right] \geq \mathbb{E}\left[\mathrm{SNR}_{10}\right] \geq \mathbb{E}\left[\mathrm{SNR}_{00}\right] \geq \mathbb{E}\left[\mathrm{SNR}_{\mathrm{iid}}\right]
$$

Thus in the high relay power regime, correlation does not hurt even when the system is ignorant of the underlying noise 


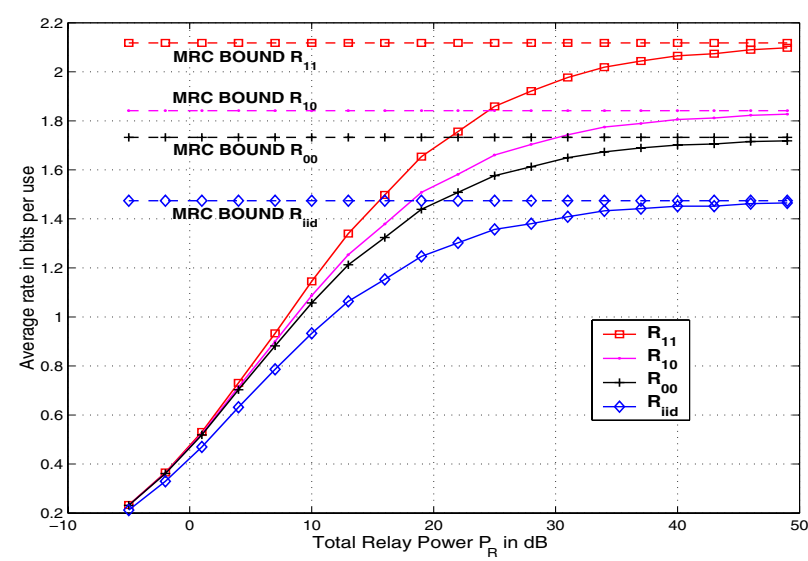

Fig. 2. Average rate of a single user system as a function of total relay power $P_{R}$ for $P=P_{I}=10$.

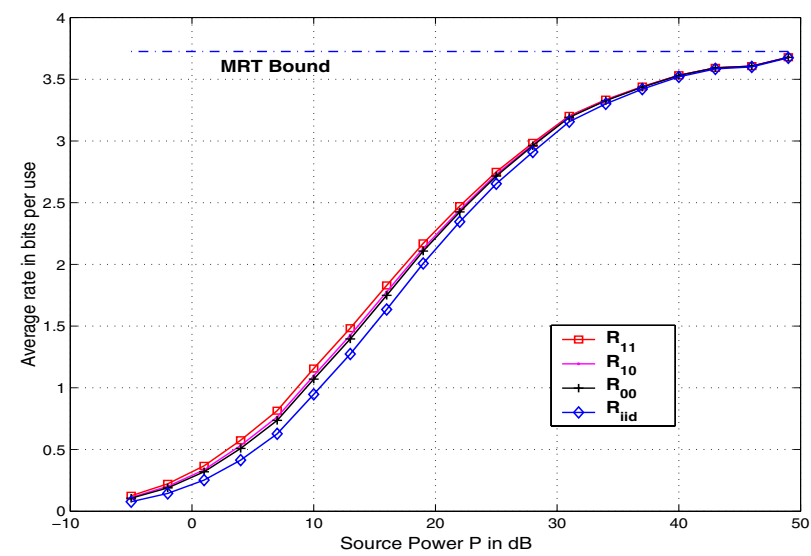

Fig. 3. Average rate of a single user system as a function of source power $P$ for $P_{R}=P_{I}=10$.

correlation structure. Importantly, it is also clear that there is a performance gain when the correlation structure is exploited.

2) Source Power $P \rightarrow \infty$ : At very high source power, the optimal relay network (Scheme-11) is equivalent to a MISO system. From (7), we have

$$
\mathrm{SNR}_{11}=P_{R} \mathbf{g}^{\dagger} \mathbf{g} .
$$

Similarly, it can be shown that the rest of the schemes also achieve the SNR in (21) at high $P$.

$$
\mathrm{SNR}_{10}=\mathrm{SNR}_{00}=\mathrm{SNR}_{\text {iid }}=P_{R} \mathbf{g}^{\dagger} \mathbf{g} .
$$

It can be noticed that this scenario represents the maximal ratio transmission (MRT) where the relays act as a multiple antenna transmitter. This is intuitive as the noise at the relays is negligible compared to the source power and therefore the effect of the noise covariance matrix is non-existent. We now verify the results with numerical analysis in the following subsection.

\section{Numerical Results}

We consider a common-interference based model to generate the relay noise covariance matrix. Each relay in addition to its thermal noise observes faded versions of common interference signals. The effective relay noise including both the interference and the local noise is given by

$$
\mathbf{n}_{\text {eff }}=\sum_{k=1}^{Q} \mathbf{h}_{\mathbf{k}} i_{k}+\mathbf{n}_{\mathbf{R}}
$$

where $i_{k}$ is the signal transmitted by the $k^{t h}$ interferer and $\mathbf{h}_{\mathbf{k}}$ is the channel between the relays and the $k^{t h}$ interferer. The covariance matrix is given by

$$
\mathbf{K}=\mathbb{E}\left[\mathbf{n}_{\text {eff }} \mathbf{n}_{\text {eff }}^{\dagger}\right]=\sum_{k=1}^{Q} \mathbf{h}_{\mathbf{k}} \mathbf{h}_{\mathbf{k}}^{\dagger} P_{I_{k}}+\mathbf{I} .
$$

The total interference power is $P_{I}=\sum_{k=1}^{Q} P_{I_{k}}$. Fig. 2 plots the average rate per channel-use as a function of the total relay power for $P=P_{I}=10$ for the case of two relays and one interferer. The main observations are

1) The performance order of the schemes in (20) which was obtained at high $P_{R}$ is valid at all values of $P_{R}$.

$2)$ The difference $\left(R_{11}-R_{10}\right)$ which indicates the benefits of learning correlation increases with relay power. With increase in relay power, the schemes diverge in performance, which is an indication that correlation impacts more at high relay power.

In Fig. 3, we plot the average rate as a function of source transmit power for $P_{R}=P_{I}=10$. Clearly, with increase in source power, the schemes achieve the MRT bound. Therefore the effect of correlation is less pronounced with increasing source power. Fig. 4 shows the average rate as a function of the interference power for one interfering node and two relays. As one can expect, the average rate decreases with $P_{I}$. However $R_{11}$ does not reach zero even at infinite interference power. This can be explained through the following: With one interfering source and $N$ relays, the average SNR for Scheme11 at very high $P_{R}$ is given by

$$
\mathbb{E}\left[\mathrm{SNR}_{11}\right]=(N-1)+\mathbb{E}\left[\frac{1}{1+P_{I}\left\|\mathbf{h}_{\mathbf{1}}\right\|^{2}}\right] .
$$

This suggests that the average SNR is at least $N-1$ irrespective of the interference power. However, the average SNR decreases when the number of interferers increases. This is due to loss in degrees of freedom due to increase in the range space of interference.

Fig. 5 shows the average sum rate versus the number of relays. The covariance matrix is generated with the help of 9 interferers with total power $P_{I}=200$. It can be noticed that the sum rate increases at a greater rate when the number of relays is greater than the number of interferers. As $P_{I} \rightarrow \infty$, $R_{11} \rightarrow 0$ when the number of relays is less than the number of interferers $(N \leq Q)$. However for $(N>Q), R_{11}$ does not vanish even when $P_{I} \rightarrow \infty$.

Remark: As we discussed earlier, the relay design problem is a tradeoff between canceling the interference and maximizing the signal power. At very high interference power, it is important to cancel the interference. At high $P_{R}$ since the network behaves as a SIMO system with $N$ antennas at the receiver, up to $(N-1)$ interfering sources can be rejected. When the number of interferers is more than the total number of relays, interference cannot be completely nulled. 


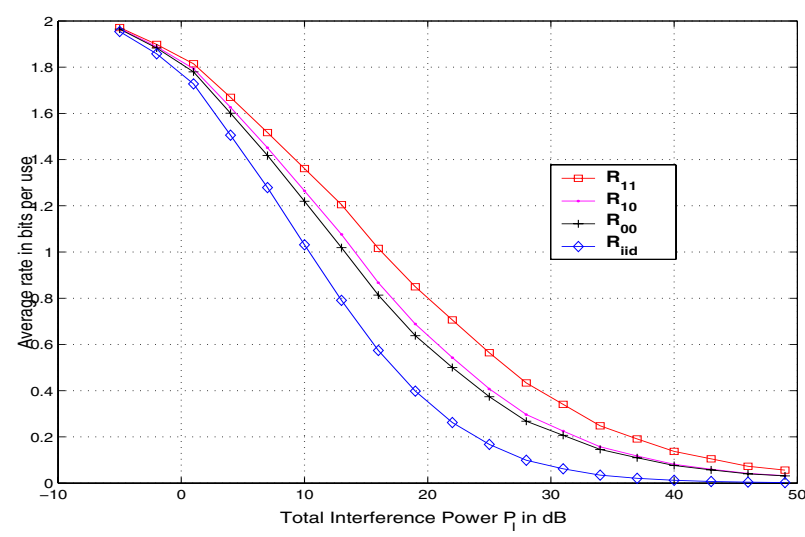

Fig. 4. Average rate of a single user system as a function of interference power $P_{I}$ for $P=10$ and $P_{R}=100$.

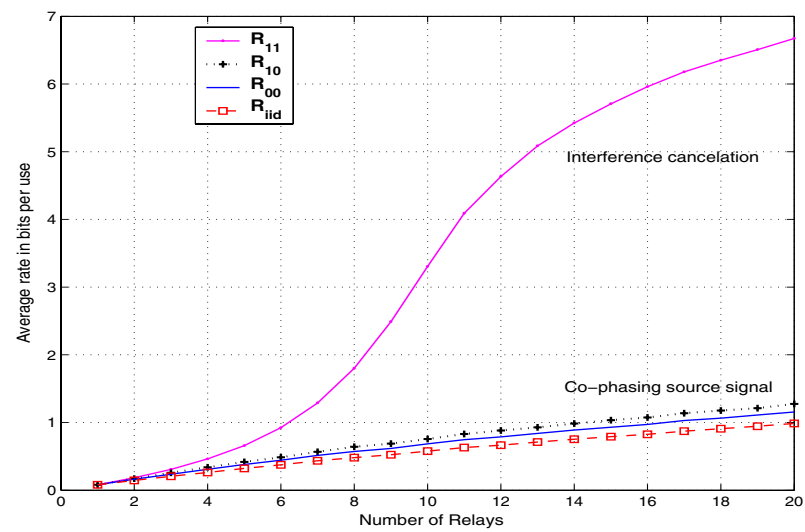

Fig. 5. Average rate of a single user system as a function of the number of relays with 9 interferers of total power $P_{I}=200(23 \mathrm{~dB})$, and $P=10$.

\section{Impact of channel knowledge at the relays}

Throughout this paper, we assume that the relays have perfect knowledge of all the channels in the network. It may seem that complete channel knowledge is required at the relays for the relationship in (20) to hold. In Scheme-11, since the relays know the noise covariance matrix, they must also know the channels between the relays and interferers (when the number of interferers is less than the number of relays). This information is not available at the relays for Scheme10. Still, it performs better than Scheme-00 where there is no noise correlation. In fact, it can be shown that Scheme10 outperforms Scheme-00 even when there is absolutely no channel knowledge at the relays including the source-relay and relay-destination channels. This is also true when the relays only have local channel information. That is, relay $R_{i}$ knows only its forward and backward channels $\mathbf{f}(i)$ and $\mathbf{g}(i)$. The following theorem states these results:

Theorem 3: The average achievable rate for a relay network is lesser with relay noise covariance matrix $\mathbf{K} \odot \mathbf{I}$ than with any general $\mathbf{K}$, where only local channel knowledge is available at the relays. In other words

$$
\mathbb{E}\left[\log \left(1+\mathrm{SNR}_{10}\right)\right] \geq \mathbb{E}\left[\log \left(1+\mathrm{SNR}_{00}\right)\right] .
$$

Further, when there is absolutely no CSI available at the relays, Scheme-10 outperforms Scheme-00 in terms of the average $\mathrm{SNR}$, i.e. $\mathbb{E}\left[\mathrm{SNR}_{10}\right] \geq \mathbb{E}\left[\mathrm{SNR}_{00}\right]$.
The proof is available in [13]. It is now clear that relay noise correlation is always helpful regardless of the channel and correlation knowledge at the relays. For maximizing signal power channel knowledge is essential while correlation knowledge is required to minimize interference power. With perfect channel knowledge, increasing the number of relays (while keeping the total relay power fixed) is helpful as signal power is increased due to coherent combining.

\section{CONCLUSION}

In this work, we considered an AF relay network wherein the relay noises are correlated which may be due to common interference or multi-hop AF relaying. We obtained closed form expressions for optimal rate maximizing relay gains and maximum achievable rate when correlation knowledge is available at the relays for both single and multi-source scenarios. Further we showed that correlation does not hurt irrespective of channel and correlation knowledge at the relays. We also showed that correlation knowledge results in significant performance improvement. Analytical and simulation results demonstrate significant rate enhancement when correlation knowledge is exploited. With appropriate gains, the relays can perform distributed interference cancelation when the number of relays is greater than the the number of interferers. The performance improvement increases with the total relay power and the number of relays. As there are significant benefits in learning correlation, practical schemes to communicate the correlation structure to the relays need to be explored.

\section{REFERENCES}

[1] H. Viswanathan and S. Mukherjee, "Throughput-range tradeoff of wireless mesh backhaul networks," IEEE Journal on Selected Areas in Communications, vol. 24, pp. 593-602, March 2006.

[2] S. A. Jafar, K. Gomadam, C. Huang, "Duality and rate optimization for AF relay MAC and BC," to appear in IEEE Transactions on Information Theory, 2007.

[3] T. M. Cover and A. El Gamaal, "Capacity Theorems for the relay channel," IEEE Transactions on Information Theory, vol. 25, no. 5, pp. 572-584, Sep 1979.

[4] G. Kramer, M. Gastpar and P. Gupta, "Cooperative Strategies and Capacity Theorems for Relay Channels," IEEE Transactions on Information Theory, vol. 51, no. 9, pp. 3037-3063, Sep 2005.

[5] J. N. Laneman, D. N. C. Tse, and G. W. Wornell, "Cooperative diversity in wireless networks: Efficient protocols and outage behavior," IEEE Trans. Inform. Theory, vol. 50, no. 12, pp. 3062-3080, 2004.

[6] K. Gomadam and S. A. Jafar, "Optimal relay functionality for SNR maximization in memoryless relay networks," IEEE Journal on Selected Areas in Communications, vol. 25, no. 2, Feb 2007.

[7] K. S. Gomadam and S. A. Jafar, "Optimizing soft information in relay networks," in Signals, Systems and Computers, 2006. ACSSC '06. Fortieth Asilomar Conference on, pp. 18-22, Oct.-Nov. 2006.

[8] P. Larsson and H. Hong, "Large scale cooperative relaying network with optimal coherernt combining under aggregate relay power constraints," in Future telecommunications conference, (Beijing, China), 2003.

[9] I. Maric and R. Yates, "Power and bandwidth allocation for cooperative strategies in Gaussian relay networks," in Proceedings of Asilomar, 2004.

[10] Z. Yi and I.-M. Kim, "Joint optimization of relay-precoders and decoders with partial channel side information in cooperative networks," Selected Areas in Communications, IEEE Journal on, vol. 25, pp. 447-458, February 2007.

[11] Y. Zhao, R. S. Adve and T. J. Lim, "Improving amplify-and-forward relay networks: optimal power allocation versus selection," To appear in IEEE Transactions on Wireless Communications, 2007.

[12] S. Berger and A. Wittneben, " Cooperative Distributed Multiuser MMSE Relaying in Wireless Ad Hoc Networks," in Asilomar Conference on Signals, Systems, and Computers, Pacific Grove, CA, 0ct 2005.

[13] K. Gomadam, S. Jafar, "The effect of noise correlation in AF relay networks," http://arxiv.org/abs/0707.1064v1. 